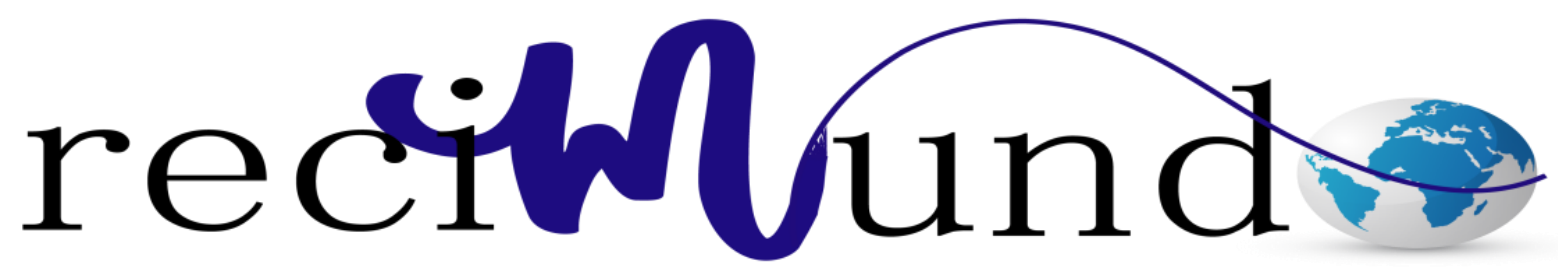

Revista Científica Mundo de la Investigación y el Conocimiento

María José Fierro Bósquez a ; Sandy Guadalupe Fierro Vasco ${ }^{\text {; }}$; Lucía Belén Aguinaga Bosquez ${ }^{\mathrm{c}}$

La Equinoterapia en niños con trastornos del espectro autista

Equine therapy in children with autism spectrum disorders

Revista Científica Mundo de la Investigación y el Conocimiento. Vol. 2 núm.3, julio, ISSN: 2588-073X, 2018, pp. 650-665

DOI: 10.26820/recimundo/2.(3).julio.2018.650-665

Editorial Saberes del Conocimiento

Recibido: 05/04/2018

Aceptado: 15/02/2018

Publicado: 30/07/2018

Correspondencia: maria.fierro@ueb.edu.ec

a. Docente Facultad de Ciencias de la Educación, Sociales, Filosóficas y Humanísticas; Universidad Estatal de Bolívar; maria.fierro@ueb.edu.ec

b. Docente Facultad de Ciencias de la Salud; Universidad Estatal de Bolívar; sandy.fierro@ueb.edu.ec

c. Docente / Coordinadora Académica Instituto Tecnológico Superior Consejo Provincial de Pichincha; lucia16_@hotmail.es 


\section{La Equinoterapia en niños con trastornos del espectro autista}

Vol. 2, núm. 3., (2018)

María José Fierro Bósquez; Sandy Guadalupe Fierro Vasco; Lucía Belén Aguinaga Bosquez

\section{RESUMEN}

Una de las principales dificultades que presentan las personas con Trastornos del Espectro Autista (TEA) es la comunicación. Asimismo, esta condición no es considerada como una enfermedad, por lo cual no tiene cura, sin embargo su tratamiento atenúa los síntomas. La presente investigación plasma los aspectos relacionados con la equinoterapia, con énfasis en la importancia de sus beneficios médicos. Para lo cual se llevó a cabo una recopilación y revisión de material documental bibliográfico que se utilizó para documentar y plasmar los mencionados aspectos. Los beneficios se extrajeron de diversos estudios del tratamiento con equinoterapia en niños con TEA, entre los principales podemos destacar: mejoras en el equilibrio, tono muscular, postura, autoestima, confianza, autocontrol emocional, tolerancia de terapias más largas, mejoras en la interacción familiar, respuesta a ordenes simples, mejoras en la atención, en el lenguaje oral y corporal, entre otros. En conclusión, la equinoterapia no es una cura para el TEA sin embargo representa una alternativa en su tratamiento que combinado con el tratamiento clínico, especialmente en los diagnósticos precoces, garantizan una mejor sociabilización del individuo y por ende, que este tenga una mejor calidad de vida.

Palabras claves: Trastornos, Espectro, Autista, Tratamiento, Equinoterapia. 


\title{
La Equinoterapia en niños con trastornos del espectro autista
}

Vol. 2, núm. 3., (2018)

María José Fierro Bósquez; Sandy Guadalupe Fierro Vasco; Lucía Belén Aguinaga Bosquez

\begin{abstract}
One of the main difficulties that people with Autism Spectrum Disorders (ASD) present is communication. Also, this condition is not considered as a disease, so there is no cure, however its treatment attenuates the symptoms. The present investigation captures the aspects related to equine therapy, with emphasis on the importance of its medical benefits. For which a compilation and review of bibliographic documentary material was carried out that was used to document and capture the aforementioned aspects. The benefits were extracted from various studies of treatment with equinotherapy in children with ASD, among the main ones we can highlight: improvements in balance, muscle tone, posture, self-esteem, confidence, emotional self-control, tolerance of longer therapies, improvements in family interaction, response to simple orders, improvements in attention, in oral and body language, among others. In conclusion, equine therapy is not a cure for ASD, however it represents an alternative in its treatment that, combined with clinical treatment, especially in early diagnoses, guarantee a better socialization of the individual and, therefore, that the patient has a better quality of life. lifetime.
\end{abstract}

Keywords: Disorders, Spectrum, Autistic, Treatment, Equine Therapy. 


\section{La Equinoterapia en niños con trastornos del espectro autista}

Vol. 2, núm. 3., (2018)

María José Fierro Bósquez; Sandy Guadalupe Fierro Vasco; Lucía Belén Aguinaga Bosquez

\section{Introducción.}

Según la Organización Mundial de la Salud, (2017) “uno de cada 160 niños tiene un trastorno del espectro autista (TEA)". Asimismo, refiere que este trastorno consiste en un “grupo de afecciones caracterizadas por algún grado de alteración del comportamiento social, la comunicación y el lenguaje, y por un repertorio de intereses y actividades restringido, estereotipado y repetitivo".

El denominado TEA no es una enfermedad, consiste en un trastorno y como tal no tiene cura, sin embargo se emplean tratamientos farmacológicos con la finalidad de disminuir los síntomas propios del trastorno tales como la ansiedad, agresividad, impulsividad, trastornos obsesivos-compulsivos, depresión, también se usa en los pacientes que presentan episodios epilépticos y existe tratamiento psicopedagógico que constituye el tratamiento central por cuanto siendo aplicado desde una temprana edad genera excelentes resultados, este incluye terapia de lenguaje, programas de socialización y estimulación sensorial múltiple. El tratamiento se basa en terapias educativas y físicas y el manejo del comportamiento de las personas que sufren el trastorno.

Una de las terapias más usadas en el tratamiento del TEA es la equinoterapia o terapia con caballos, en virtud de lo cual es importante mencionar que el uso de este noble animal con fines terapéuticos data de muchos años atrás. 


\section{La Equinoterapia en niños con trastornos del espectro autista}

Vol. 2, núm. 3., (2018)

María José Fierro Bósquez; Sandy Guadalupe Fierro Vasco; Lucía Belén Aguinaga Bosquez

"Muchos pueblos entendieron que el caballo era más que un animal a su servicio para la guerra, el transporte y el trabajo. En algunas culturas lo elevaron al estatus de divinidad. Durante milenios, estos animales han demostrado a los humanos que su presencia y compañía causan grandes cambios anímicos. Sin embargo, su función terapéutica nunca había sido objeto de estudio de la ciencia, hasta mediados del siglo XX, cuando empezó a utilizárselos para rehabilitación de diferentes enfermedades que involucraban discapacidad física y psíquica”. (Buscaglia, 2015)

"El caballo es un animal sensible y grande, pero en absoluto peligroso. Es sin duda un animal noble, hermoso y con una sensibilidad especial: son capaces de entender nuestros sentimientos sin hablar, razón por la cual, los niños encontrarán en el caballo una aceptación especial, ejercicio y una relación muy distinta a la habitual: la compenetración, básica entre jinete y caballo”. (García, 2018)

Existen muchas aplicaciones de la equinoterapia en líneas generales, entre los que se pueden destacar las aplicaciones para problemas físicos tales como, "problemas del equilibrio y de la coordinación dinámica, problemas posturales, del tono y la flexibilidad muscular, asimetrías musculares del tronco y la cadera y para mejorar el desarrollo motor grueso de las personas con dificultades, entre otros...". Por otra parte, se encuentran los beneficios mentales, aplicándose la terapia en "problemas de socialización y con trastornos de conducta; problemas en chicos con riesgo de exclusión social; en trastornos por déficit atencionales; en el incremento de las conductas comunicativas y de interacción en chicos con autismo, entre otros...”. (García Peña \& García Gómez, 2015) 


\section{La Equinoterapia en niños con trastornos del espectro autista}

Vol. 2, núm. 3., (2018)

María José Fierro Bósquez; Sandy Guadalupe Fierro Vasco; Lucía Belén Aguinaga Bosquez

Se trata de un tratamiento en donde el individuo se beneficia del contacto con el animal y la calma del medio ambiente donde la práctica, además de servir como vehículo para el empleo de otros tratamientos como la fisioterapia.

Pérez y otros, (2013) mencionan que "La equinoterapia no constituye la cura del autismo, pero si una oportunidad inigualable para ofrecerle al niño actividades científicamente organizadas, orientadas a estimular su desarrollo y contacto con el medio". Asimismo, hacen mención de que el autismo de concepto es una enfermedad para toda la vida, y citan a Helt (2008), Siller (2008), Magiati (2007), al destacar que han demostrado sin lugar a duda que una adecuada estimulación especialmente precoz permite el desarrollo de habilidades que mejoran el desempeño funcional del niño autista.

Es importante destacar que "La equinoterapia abarca la integración de cuatro ámbitos profesionales diferentes: la medicina, la psicología, la pedagogía y el deporte. El área médica desempeña un papel dominante en la Hipoterapia porque la monta a caballo se utiliza a manera de fisioterapia, indicada para pacientes con disfunciones neuromotoras de origen neurológico, traumático o degenerativo”. (Coronel Chávez \& León Franco, 2013, p. 22)

En tal sentido, la presente investigación reúne los aspectos básicos relacionados con el TEA como preámbulo a su tratamiento médico con equinoterapia. A pesar de la íntima relación entre las áreas relacionadas con el tratamiento con caballos (la psicología, psicopedagogía, deporte y medicina) nos apegaremos al tratamiento médico, cuya importancia se fundamenta en 


\section{La Equinoterapia en niños con trastornos del espectro autista}

Vol. 2, núm. 3., (2018)

María José Fierro Bósquez; Sandy Guadalupe Fierro Vasco; Lucía Belén Aguinaga Bosquez

la recopilación de opiniones y estudios, los cuales sirven de apoyo para la difusión de esta terapia por cuanto representa un avance para los pacientes con esta condición, que se centra en mejorar su calidad de vida.

\section{Materiales y Métodos.}

La presente investigación se llevó a cabo a través de una recopilación y revisión de material documental bibliográfico que se utilizó para documentar y plasmar aspectos básicos relacionados con el TEA y su tratamiento médico basado en la equinoterapia y sus beneficios para los pacientes con esta condición.

Según Tancara (1993), la investigación documental es “una serie de métodos y técnicas de búsqueda, procesamiento y almacenamiento de la información contenida en los documentos, en primera instancia, y la presentación sistemática, coherente y suficientemente argumentada de nueva información en un documento científico, en segunda instancia” (p. 94)

\section{Resultados.}

\section{Trastornos del Espectro Autista - TEA (definición)}

"Los trastornos del espectro autista (TEA) son un grupo de complejos trastornos del desarrollo cerebral. Este término genérico abarca afecciones tales como el autismo, el trastorno desintegrador infantil y el síndrome de Asperger. Estos trastornos se caracterizan por dificultades en la comunicación y la interacción social y por un 


\section{La Equinoterapia en niños con trastornos del espectro autista}

Vol. 2, núm. 3., (2018)

María José Fierro Bósquez; Sandy Guadalupe Fierro Vasco; Lucía Belén Aguinaga Bosquez repertorio de intereses y actividades restringido y repetitivo". (Organización Mundial de la Salud, 2016)

\section{Equinoterapia (definición)}

De la Prieta Cuervo, (2017) en su trabajo cita a García (2010) y Maciques (2013) para definir la equinoterapia como una "terapia integral empleada por profesionales que utiliza al caballo y las técnicas ecuestres como instrumento terapéutico para la rehabilitación en personas con deficiencias mentales, físicas, sociales y temperamentales”. (p. 20)

\section{Divisiones}

Castillo, (2011) cita la subdivisión del Congreso de Equitación Terapéutica de Toronto (Canadá) 1988, la cual fue aprovechada posteriormente a nivel internacional por la FRDI (Federation of Riding forthe Disabled International) con sede en Bélgica:

\section{La hipoterapia}

La hipoterapia está destinada a personas que por su grave discapacidad física y/o cognitiva no puede ejercer prácticamente ninguna acción sobre el caballo. En la mayoría de los casos esta se lleva a cabo sin montura a los fines de mantener un contacto más directo entre el paciente y el caballo. 


\section{La Equinoterapia en niños con trastornos del espectro autista}

Vol. 2, núm. 3., (2018)

María José Fierro Bósquez; Sandy Guadalupe Fierro Vasco; Lucía Belén Aguinaga Bosquez

\section{Equitación terapéutica.}

Esta actividad está destinada a personas que pueden ejercer alguna acción sobre el caballo, a diferencia de la anterior, ya sea durante la higiene, preparación del material o a la hora de montar a caballo. Mediante algunas adaptaciones individualizadas de acuerdo al tipo de discapacidad, se enseña a montar a caballo a personas discapacitadas teniendo la actividad un impacto favorable en especial en el área psicológica, pedagógica y social del alumno. Los objetivos terapéuticos son más generales, el paciente monta generalmente sólo, con o sin silla y aprende los principios básicos de la equitación, paso, trote y galope según los avances del paciente.

\section{Equitación adaptada}

“Destinada a personas que practican la equitación con un objetivo lúdico o deportivo pero que por su discapacidad necesitan adaptaciones para acceder o manejar el caballo: rampas, escaleras, monturas adaptadas, estribos o riendas adaptadas, entre otros".

\section{Equinoterapia social}

Término utilizado por algunos autores para hacer referencia a la "actividad en el manejo de problemas de integración social, alcoholismo, drogadicción, entre otros, mediante el uso del caballo como elemento de unión y marcador de un objetivo común que fomente la relación y el trabajo en equipo". 


\section{La Equinoterapia en niños con trastornos del espectro autista}

Vol. 2, núm. 3., (2018)

María José Fierro Bósquez; Sandy Guadalupe Fierro Vasco; Lucía Belén Aguinaga Bosquez

\section{Volteo Terapéutico}

De acuerdo al autor Serrano (2007) esta modalidad "orienta psicopedagógicamente e interviene en el desarrollo de conductas psicosociales positivas. Se consigue desarrollar la

psicomotricidad, la capacidad de atención y de concentración y la cognición”. (De la Prieta Cuervo, 2017, p. 25)

\section{Principios terapéuticos activos durante la monta}

Coronel Chávez \& León Franco (2013) mencionan tres estímulos que el caballo es capaz de trasmitir durante la monta:

$\checkmark \quad$ Impulsos rítmicos:

Son transmitidos por el lomo y el costado del caballo, ya que cada movimiento de sus miembros posteriores y anteriores provoca contracciones y extensiones musculares en el músculo dorsal y lumbar de su lomo y en los músculos ventrales del costado. Los músculos se contraen y distienden de manera alternada de lado a lado en el paso y el trote. Investigaciones sobre la equinoterapia indican que el paso se trasmite aproximadamente de 90 a 110 impulsos por minuto a la pelvis del jinete, los cuales aumentan en cantidad e intensidad en el trote. Estos impulsos fisiológicos se propagan hacia arriba por medio de la columna vertebral hasta la cabeza provocando en el jinete las 


\section{La Equinoterapia en niños con trastornos del espectro autista}

Vol. 2, núm. 3., (2018)

María José Fierro Bósquez; Sandy Guadalupe Fierro Vasco; Lucía Belén Aguinaga Bosquez

reacciones de enderezamiento y equilibrio ya mencionadas, activadas por las compensaciones corporales y propiciando una neuroestimulación cerebral.

$\checkmark \quad$ Calor corporal:

"En virtud de que el cuerpo del caballo alcanza hasta $38,8^{\circ} \mathrm{C}$ durante su movimiento se aprovecha como un instrumento calorífico para distender y relajar musculatura, ligamentos, estimular la sensopercepción táctil. El paciente monta sin albardón y si es posible a pelo. Esto permite que el calor del animal se transmita al cinturón pélvico y a los miembros inferiores del paciente, lo que favorece en gran medida el relajamiento de los músculos de las piernas y glúteos. Se ha comprobado que con el contacto y el calor del caballo se adquiere un notable afecto distensor de músculos y ligamientos así como una prolongada duración de esta distensión (hasta 6 horas en aductores)". (Asociación de Equinoterapia Ismael Pinto, s.f.)

$\checkmark \quad$ Patrón de marcha:

“El valor fisioterapéutico, por la transmisión de un patrón de locomoción tridimensional equivalente al patrón fisiológico de la marcha humana, de esta forma permite trabajar con personas que carecen de la facultad de caminar y podrán desarrollar la coordinación y la estabilización del tronco y de la cabeza. Este principio depende en sumo grado del movimiento correcto del caballo; un paso rítmico y regular, y un adecuado trabajo muscular del lomo”. (Asociación de Equinoterapia Ismael Pinto, s.f.) 


\section{La Equinoterapia en niños con trastornos del espectro autista}

Vol. 2, núm. 3., (2018)

María José Fierro Bósquez; Sandy Guadalupe Fierro Vasco; Lucía Belén Aguinaga Bosquez

\section{Beneficios de la equinoterapia en el tratamiento médico del TEA}

"La equinoterapia ha demostrado su eficacia para mejorar el equilibrio y la movilidad, y por esto se ha empleado en personas que sufren diferentes tipos de parálisis. Asimismo, tiene efectos sobre la comunicación y el comportamiento, y puede mejorar la calidad de vida de personas con necesidades especiales. La equinoterapia es considerada por el paciente como una actividad recreativa, que se realiza en un entorno agradable que ayuda a relajarse”. (González Núñez, 2018)

Al respecto Macías (2018) acota que la equinoterapia trae beneficios para el paciente tales como: "mejora las habilidades de comunicación, su funcionamiento psicológico, cognitivo y conductual, las habilidades motoras y su coordinación, la integración sensorial, su tono muscular, su control del tronco, control de su postura, su fuerza y su acondicionamiento cardiovascular".

En un estudio realizado por Coronel Chávez \& León Franco (2013) a 20 niños autistas, entre 6 y 10 años, de ambos sexos, del Centro de Rehabilitación Infantil de la Secretaría de la Defensa Nacional, durante los meses de Mayo a Julio de 2012, aplicando tratamiento con equinoterapia, obtuvo y superó los resultados esperados del $25 \%$ de mejoría en el desempeño funcional de los pacientes evaluados en base al seguimiento de órdenes simples por medio de la equinoterapia, 19 de los 20 niños (indistintamente del sexo), realizaron actividades en casa tras una sola orden e incrementaron la socialización con los miembros de la familia. Asimismo, dicha 


\section{La Equinoterapia en niños con trastornos del espectro autista}

Vol. 2, núm. 3., (2018)

María José Fierro Bósquez; Sandy Guadalupe Fierro Vasco; Lucía Belén Aguinaga Bosquez

mejoría se vio reflejada en las sesiones de terapia que toman los niños después en dicho centro, obteniendo un mejor desempeño y tolerando sesiones más largas.

Este estudio fue concluyente en cuanto a la mejora significativa de la equinoterapia en niños con autismo, los resultados repercutieron en mejoras en las áreas tanto terapéuticas como familiares, en cuanto a la tolerancia de sesiones terapéuticas más largas, seguimiento de órdenes, mejoras en el comportamiento e interacción.

Asimismo, "de acuerdo a registros obtenidos por la North American Riding for the Handicap Asociation y el National Center for the Equine Facilite Teraphy, por medio del uso de radiografías, electromiografías y transferencias del movimiento tambaleante del caballo al paciente-alumno mediante películas científicas, se detecta que el movimiento rítmico y continuo del paso del caballo permite al cerebro a través de la médula registrar músculos, órganos y envíe señales a éstos, para que se inicie el proceso de estimulación neuro-muscular. En este proceso de neuro-transmisión de la sinapsis de la neurona se promueven la generación de procesos mentales, liberándose productos químicos, como serotonina, dopamina, endorfinas entre otras, que al ser liberados producen sensación de placer y además le permite al cerebro registrar zonas que de otra manera serían más difíciles, este mecanismo es importante ya que mientras el sujeto está realizando la actividad arriba del caballo, disfrutando del medio natural su sistema nervioso central está trabajando en las condiciones mencionadas”. (Coronel Chávez \& León Franco, 2013) 


\section{La Equinoterapia en niños con trastornos del espectro autista}

Vol. 2, núm. 3., (2018)

María José Fierro Bósquez; Sandy Guadalupe Fierro Vasco; Lucía Belén Aguinaga Bosquez

De la Prieta Cuervo (2017) en un estudio realizado a 4 niños con autismo en el Centro Hípico "El Sable", en Laredo, durante 10 sesiones de equinoterapia de una hora, una vez a la semana, a través de la cual concluyó citando a los autores Falke (2009) y Woolfolk (1985) "la equinoterapia combinada con la terapia clínica es una alternativa muy eficaz y efectiva para tratar el Trastorno del Espectro Autista (TEA)”. (p. 36)

Durante este estudio la autora pudo observar mejoras en los niños tales como cumplir órdenes, mantener la memoria con respecto a las órdenes, mayor disposición para llevar a cabo las instrucciones, la montada y desmontada por cuenta propia, aumentaron la capacidad de mantener una conversación con otras personas, mejoraron la capacidad de mantener la mirada y el autocontrol ante sus propias emociones, igualmente mejoraron el contacto físico con el caballo e incrementado sus habilidades y destrezas para resolver algunos problemas.

\section{Conclusiones.}

Dentro de los beneficios de la equinoterapia tenemos los físicos tales como la mejora de la postura, del tono muscular, mejora del sistema nervioso central, otros psicoterapéuticos como mejora en la memoria, retención y cumplimiento de órdenes, mejoras en el lenguaje oral y corporal, mejor disposición y tolerancia para las terapias, mejoras en la interacción familiar y con el equipo terapeuta, mejoras en la atención y el sostenimiento de la mirada, autocontrol de las emociones, mejoras en la conversación con otras personas, entre las principales. 


\section{La Equinoterapia en niños con trastornos del espectro autista}

Vol. 2, núm. 3., (2018)

María José Fierro Bósquez; Sandy Guadalupe Fierro Vasco; Lucía Belén Aguinaga Bosquez

Es innegable la repercusión positiva que tiene la terapia de caballos en los niños con TEA, sin embargo, esta terapia aunada a otras terapias clínicas es una alternativa eficaz y efectiva en el tratamiento del trastorno.

En conclusión, la equinoterapia en el tratamiento de niños con TEA no es una cura, sin embargo representa una gran posibilidad en la atenuación de los síntomas, lo ideal es combinar las diferentes disciplinas relacionadas con este tratamiento dada su estrecha relación y el aprovechamiento que puede tener la aplicación de una sobre la otra, todo ello con el objeto de incluir al niño con este trastorno en las relaciones interfamiliares y sociales, y por ende, garantizarles una mejor calidad de vida.

\section{Bibliografía.}

Asociación de Equinoterapia Ismael Pinto. (s.f.). Asociación de Equinoterapia Ismael Pinto. $\begin{array}{llllll}\text { Recuperado el } 03 \text { de } & \text { Agosto de }\end{array}$ http://www.asocequinoterapia.org/Principios/Principios.htm

Buscaglia, T. S. (12 de Enero de 2015). La Nación. Recuperado el 03 de Agosto de 2018, de https://www.lanacion.com.ar/1759354-caballos-que-sanan-la-equinoterapia-unaalternativa-para-curar

Castillo, M. d. (2011). UCA. Recuperado el 03 de Agosto de 2018, de http://actividades.uca.es/compromisoambiental/2011/C02/3

Coronel Chávez, J. M., \& León Franco, J. (2013). Universidad Autónoma del Estado de México. $\begin{array}{llllll}\text { Recuperado el } 03 \text { de } & \text { Agosto de }\end{array}$ http://ri.uaemex.mx/bitstream/handle/20.500.11799/14298/404420.pdf?sequence=1

De la Prieta Cuervo, J. (27 de Junio de 2017). Universidad de Cantabria. Recuperado el 03 de Agosto de 2018, de https://repositorio.unican.es/xmlui/bitstream/handle/10902/11839/PrietaCuervoJazminaD eLa.pdf? sequence $=1$ 


\section{La Equinoterapia en niños con trastornos del espectro autista}

Vol. 2, núm. 3., (2018)

María José Fierro Bósquez; Sandy Guadalupe Fierro Vasco; Lucía Belén Aguinaga Bosquez

García Peña, I. M., \& García Gómez, A. (07 de Abril de 2015). Autismo Diario. Recuperado el 03 de Agosto de 2018, de https://autismodiario.org/2015/04/07/caballos-y-terapia-dellenguaje/

García, M. (19 de Marzo de 2018). Experto Animal. Recuperado el 03 de Agosto de 2018, de https://www.expertoanimal.com/equinoterapia-para-ninos-autistas-2318.html

González Núñez, H. (02 de Agosto de 2018). Webconsultas. Recuperado el 03 de Agosto de 2018, de https://www.webconsultas.com/autismo/terapias-con-animales-para-el-autismo3128

Macias, F. (2018). ¿Qué es el autismo? Recuperado el 03 de Agosto de 2018, de https://queeselautismo.com/hipoterapia-y-autismo/

Organización Mundial de la Salud. (Abril de 2016). Organización Mundial de la Salud. Recuperado el 03 de Agosto de 2018, de http://www.who.int/features/qa/85/es/

Organización Mundial de la Salud. (04 de Abril de 2017). Organización Mundial de la Salud. Recuperado el 03 de Agosto de 2018, de http://www.who.int/es/news-room/factsheets/detail/autism-spectrum-disorders

Pérez, L., Pérez Guerrero, M., Santisteban, M., Salas, D., Sólis, X., \& Chirino, L. (2013). La equinoterapia en la rehabilitación integral del autismo. EFdeportes.com(176). Recuperado el 03 de Agosto de 2018, de http://www.efdeportes.com/efd176/laequinoterapia-en-la-rehabilitacion-del-autismo.htm

Tancara, C. (1993). La Investigación Documental. Temas Sociales(17), 91-106. Obtenido de http://www.revistasbolivianas.org.bo/pdf/rts/n17/n17a08.pdf 